\title{
Targeting interleukins in chronic airway diseases using advanced drug delivery
}

\author{
Saurabh Satija $a^{\ddagger 1,2(\mathbb{D})}$, Meenu Mehta $a^{\ddagger 1,3(\mathbb{D}), G a u r a v}$ Gupta $^{4}$ (D), Dinesh Kumar Chellappan ${ }^{5}$ (D) \\ \& Kamal Dua*,1,3,6,7 iD \\ ${ }^{1}$ Discipline of Pharmacy, Graduate School of Health, University of Technology Sydney, NSW 2007, Australia \\ ${ }^{2}$ School of Pharmaceutical Sciences, Lovely Professional University, Phagwara, Punjab, 144411, India \\ ${ }^{3}$ Centre for Inflammation, Centenary Institute, Sydney, NSW 2050, Australia \\ ${ }^{4}$ School of Pharmacy, Suresh Gyan Vihar University, Jagatpura, Jaipur, 302017, India \\ ${ }^{5}$ Department of Life Sciences, School of Pharmacy, International Medical University, Bukit Jalil, Kuala Lumpur, 57000, Malaysia \\ ${ }^{6}$ Priority Research Centre for Healthy Lungs, University of Newcastle \& Hunter Medical Research Institute, Lot 1 Kookaburra Circuit, \\ New Lambton Heights, Newcastle, NSW 2305, Australia \\ ${ }^{7}$ School of Pharmaceutical Sciences, Shoolini University, Solan, Himachal Pradesh, 173229, India \\ *Author for correspondence: Kamal.Dua@uts.edu.au \\ $\ddagger$ Authors contributed equally
}

“Interleukins are evolving treatment strategies in managing the chronic respiratory diseases inflammation, which can be effectively target in managing disease pathology using the concept of advanced drug delivery systems."

First draft submitted: 9 June 2020; Accepted for publication: 24 July 2020; Published online: 5 October 2020

Keywords: advanced drug delivery $\bullet$ chronic airway diseases $\bullet$ cytokines $\bullet$ interleukins

Chronic airway diseases are categorized as a heterogeneous group of diseases with multiple phenotypes, that are primarily based on the existence of inflammatory cell infiltration mechanisms. Interleukins are key players in the pathogenesis of inflammation and in the propagation of chronic inflammatory airway diseases, such as, chronic obstructive pulmonary disease (COPD), bronchiectasis, cystic fibrosis, lung cancer and allergic asthma [1]. Interleukins are cytokines that are produced predominantly by the $\mathrm{T}$ cells. Understanding their mechanisms and their roles in pathogenesis have increased their potential as possible targets for new treatments in chronic airway diseases, employing advanced drug delivery methods [2].

In the cytokine family, interleukins form a major class of cell signaling molecules. Previously, they were thought to be proteins, that were produced by leucocytes. It has now become apparent that they are formed by different cell types with overlapping properties of pleiotropic nature. Helper $\mathrm{CD} 4^{+} \mathrm{T}$ lymphocytes, monocytes, macrophages and endothelial cells produce most of the known interleukins. While interleukins are often named individually, their families frequently include multiple groups. There are a total of 37 interleukins that are involved in major chronic airway diseases like asthma and COPD [3].

Asthma, according to a recent study, may comprise of several forms, including allergic asthma and steroid-proof asthma. It is well known that allergens activate mast cells, which lead to the inflammation of the airways, triggering an acute inflammatory response in susceptible individuals. Dendritic cells that drive differentiation and secretion of Th2 cytokines, such as IL-4, IL-5, IL-9, IL-13 and IL-17 recognize the allergens. Among these cytokines, IL-4 and IL-13 cause B cells to generate IgE, whereas, IL-13 induces the secretion of periostin by epithelial cells [4]. Furthermore, IL-9 causes hypersecretion of mucus by inducing the mast cells. IL-5 supports the development of eosinophils, their recruitment and their survival. IL-17 acts on bronchial epithelial cells, whereby it stimulates the release of CXCL8. These interleukins are also linked to airway hyper-responsiveness and remodeling [5].

COPD is attributed primarily to smoking, or any prolonged substantial exposure to smoke, dust or polluted air, which eventually result in a range of pathological complications that include, chronic bronchial inflammation, lung tissue damage and minor scarring. Cytokines, namely, TNF- $\alpha$, IFN- $\gamma$, IL-1, IL-6, IL-8, IL-18 and IL-32 play a major role in the activation of various inflammatory processes that involve, T cells, neutrophils and macrophages [3]. In 
addition, there are several studies that have reported the role of IL-17 in the pathogenesis of COPD [6]. Interleukins have generated tremendous interest lately and have formed an integral part of evolving novel treatment strategies that include the use of advanced drug delivery systems, in the management of inflammatory chronic respiratory diseases.

\section{Need for advanced drug delivery strategies}

Interleukins are evolving treatment strategies in managing the chronic respiratory diseases inflammation, which can be effectively target in managing disease pathology using the concept of advanced drug delivery systems. The conventional pharmacotherapy for chronic airway diseases can be divided into several categories depending on the type of therapeutic agent used. There are various chemical compounds, peptides, antibodies and genetic factors that are employed in the treatment of chronic airway diseases (e.g., siRNA, shRNA and miRNA) [7]. Unfortunately, pharmacotherapy alone cannot completely and effectively cure chronic airway diseases. The only existing treatment strategy adopted for asthma is the management of the symptoms. In addition, treatment strategies for COPD currently involve, steroids, bronchodilators, pirfenidone and nintedanib; nevertheless, there are no effective treatment modalities available for complete cure [8].

Emerging advanced nanoscale drug delivery strategies have shown promising results in the pharmacotherapy of chronic respiratory diseases. Such advanced pharmaceuticals may boost the pharmacokinetic profile of loaded therapy, based on their intrinsic physical properties. In addition, recent developments in nanocarriers with different targeting motives may mitigate the impact of drugs on selective delivery to target cells $[9,10]$. Traditional drug therapies often have limitations. The pharmacokinetic profile and low diffusion rates of conventional drug therapies often result in a low therapeutic response. For example, in gene therapy, an efficient vector system is often required. Apparently, gene molecules are not easily delivered in cells without carriers and are often degraded to biological fluids. To overcome these issues, nano delivery devices have come up as promising carriers in recent decades [11,12].

\section{Advanced drug delivery strategies that target interleukins}

Recent reports have emphasized the potential applications of novel drug delivery systems in targeting chronic airway diseases. Yong et al. demonstrated that quercetin loaded liquid crystalline nanoparticles (LCNs) may be a therapeutic treatment for asthma, as important pro-inflammatory markers such as IL-1 $\beta$, IL-6 and IL-8 have been successfully suppressed and thus the anti-inflammatory activity of the drug may be significantly enhanced. The improved anti-inflammatory activity of quercetin LCNs may likely be attributed to the properries of LCNs themselves, which are bioadhesive in nature. They enhance the permeation of broncho-epithelial cell membranes resulting in increased cellular uptake and drug absorption [13]. Another study reported the effectiveness of liposomal curcumin in asthma at lower doses when compared with free curcumin. The combination effect of curcumin and phospholipids had enabled the curcumin liposomes to suppress the pro-inflammatory markers (IL-6, IL-8, IL-1 $\beta$ and TNF- $\alpha$ ) in the BCi-NS1.1 cell line [14].

Several other studies have reported that the nanoparticle-based delivery of various drugs such as, dexamethasone, andrographolide and budesonide has allowed a more effective inhibitory effect of Th2 cytokines, mainly, IL4 and IL-13, possibly because of enhanced bioavailability, better lung deposition and sustained release of the drug [15-17]. Luo et al. have previously assessed the implications of nanovaccine with poly(lactic-co-glycolic) acid (PLGA-ovalbumin [OVA] + A20 [ubiquitin E3 ligases]) on asthma allergy in a murine model. The study showed that, the nanovaccine significantly increased the generation of Treg cell and IL-10, and eventually decreased airway allergic response in sensitized mice [18]. Recent studies have further shown that silica dioxide and zinc oxide nanoparticles have negative impacts on asthma exacerbation as they substantially increase mRNA expression levels of inflammatory cytokines followed by an elevation in TXNIP, NLRP3 and IL-1 $\beta$-proteins $[19,20]$.

\section{Conclusion}

Chronic inflammation is a global issue and is largely advancing due to an aging population and a substantial rise in air pollution from particulate matter over recent decades. Developing a more precise understanding of pro- and anti-inflammatory cytokines that regulate and/or facilitate disease severity is important for developing advanced therapeutics. Given the substantial progress in chronic inflammatory airway diseases, most of our clinical strategies result in serious side effects. In this commentary, we have addressed advanced pharmaceutical drug therapies for chronic airway diseases. Although, clinical applications of nanocarriers are still in their early stages; their applications in the field of respiratory medicine and innovative nanomaterials are continuously being expedited. Despite the 
primary challenges, there may be highly reliable advanced options that deliver medical sciences and technological advances in nanobiotechnology, which may lead to the effective management of chronic airway diseases.

\section{Financial \& competing interests disclosure}

M Mehta is supported by the Graduate School of Health and University of Technology Sydney (International Research Training Program Scholarship). K Dua is supported by a project grant from Rebecca L Cooper Medical Research Foundation and Sydney Partnership for Health, Education, Research and Enterprise. The authors have no other relevant affiliations or financial involvement with any organization or entity with a financial interest in or financial conflict with the subject matter or materials discussed in the manuscript apart from those disclosed.

No writing assistance was utilized in the production of this manuscript.

\section{References}

1. Chung KF. Targeting the interleukin pathway in the treatment of asthma. Lancet 386(9998), 1086-1096 (2015).

2. Garth J, Barnes JW, Krick S. Targeting cytokines as evolving treatment strategies in chronic inflammatory airway diseases. Int. J. Mol. Sci. 19(11), 3402 (2018).

3. Atamas SP, Chapoval SP, Keegan AD. Cytokines in chronic respiratory diseases. F1000 Biol. Rep. 5(3), doi:10.3410/B5-3 (2013).

4. Zhu J, Paul WE. CD4 T cells: fates, functionsand faults. Blood 112(5), 1557-1569 (2008).

5. Kim EY, Battaile JT, Patel AC et al. Persistent activation of an innate immune response translates respiratory viral infection into chronic lung disease. Nat. Med. 14(6), 633-640 (2008).

6. Doe C, Bafadhel M, Siddiqui S et al. Expression of the T helper 17-associated cytokines IL-17A and IL-17F in asthma and COPD. Chest 138(5), 1140-1147 (2010).

7. Mehta M, Dhanjal DS, Paudel KR et al. Cellular signalling pathways mediating the pathogenesis of chronic inflammatory respiratory diseases: an update. Inflammopharmacology 28, 795-817 (2020).

8. Dua K, Malyla V, Singhvi G et al. Increasing complexity and interactions of oxidative stress in chronic respiratory diseases: an emerging need for novel drug delivery systems. Chem. Biol. Interact. 1(299), 168-178 (2019).

9. Dua K, Wadhwa R, Singhvi G et al. The potential of siRNA based drug delivery in respiratory disorders: recent advances and progress. Drug Dev. Res. 80(6), 714-730 (2019).

10. Wadhwa R, Pandey P, Gupta G et al. Emerging complexity and the need for advanced drug delivery in targeting Candida species. Curr. Top. Med. Chem. 19(28), 2593-2609 (2019).

11. Thakur AK, Chellappan DK, Dua K, Mehta M, Satija S, Singh I. Patented therapeutic drug delivery strategies for targeting pulmonary diseases. Expert Opin. Ther. Pat. 30(5), 375-387 (2020).

12. Dua K, Rapalli VK, Shukla SD et al. Multi-drug resistant mycobacterium tuberculosis \& oxidative stress complexity: emerging need for novel drug delivery approaches. Biomed. Pharmacother. 107, 1218-1229 (2018).

13. Cherk Yong DO, Saker SR, Wadhwa R et al. Preparation, characterization and in-vitro efficacy of quercetin loaded liquid crystalline nanoparticles for the treatment of asthma. J. Drug Deliv. Sci. Technol. 54, doi:10.1016/j.jddst.2019.101297 (2019).

14. Ng ZY, Wong JY, Panneerselvam J et al. Assessing the potential of liposomes loaded with curcumin as a therapeutic intervention in asthma. Colloids Surf. B Biointerfaces 172, 51-59 (2018).

15. Kenyon NJ, Bratt JM, Lee J et al. Self-assembling nanoparticles containing dexamethasone as a novel therapy in allergic airways inflammation. PLoS ONE 8(10), doi:10.1371/journal.pone.0077730 (2013).

16. Chakraborty S, Ehsan I, Mukherjee B et al. Therapeutic potential of andrographolide-loaded nanoparticles on a murine asthma model. Nanomed. Nanotechnol. Biol. Med. 20, doi:10.1016/j.nano.2019.04.009 (2019).

17. Ahmad N, Ahmad R, Almakhamel MZ et al. A comparative pulmonary pharmacokinetic study of budesonide using polymeric nanoparticles targeted to the lungs in treatment of asthma. Artif. Cells Nanomed. Biotechnol. 48(1), 749-762 (2020).

18. Luo XQ, Zhong JW, Qiu SY et al. A20-OVA nanoparticles inhibit allergic asthma in a murine model. Inflammation 43, 953-961 (2020).

19. Ko JW, Shin NR, Je-Oh L et al. Silica dioxide nanoparticles aggravate airway inflammation in an asthmatic mouse model via NLRP3 inflammasome activation. Regul. Toxicol. Pharmacol. doi:10.1016/j.yrtph.2020.104618 112 (2020).

20. Larsen ST, Jackson P, Poulsen SS et al. Airway irritation, inflammation, and toxicity in mice following inhalation of metal oxide nanoparticles. Nanotoxicology 10(9), 1254-1262 (2016). 
